\title{
Neurologic manifestations in 1760 COVID-19 patients admitted to Papa Giovanni XXIII Hospital, Bergamo, Italy
}

\author{
Nicola Rifino ${ }^{1}$ - Bruno Censori ${ }^{2,4} \cdot$ Emanuela Agazzi $^{2} \cdot$ Dario Alimonti $^{1,2} \cdot$ Virginio Bonito $^{2} \cdot$ Giorgia Camera $^{2}$. \\ Marta Zaffira Conti ${ }^{2} \cdot$ Camillo Foresti $^{2} \cdot$ Barbara Frigeni $^{2} \cdot$ Simonetta Gerevini $^{3} \cdot$ Maria Grimoldi $^{2} \cdot$ Sara La Gioia $^{2}$. \\ Tania Partziguian ${ }^{2}$. Stefano Quadri ${ }^{2} \cdot$ Riccardo Riva $^{2} \cdot$ Maria Cristina Servalli ${ }^{2} \cdot$ Manlio Sgarzi ${ }^{2} \cdot$ Benedetta Storti $^{1}$. \\ Marcella Vedovello ${ }^{2}$ Elisabetta Venturelli ${ }^{2} \cdot$ Martina Viganò $^{1} \cdot$ Annapaola Callegaro $^{5} \cdot$ Marco Arosio $^{5}$. \\ Maria Sessa² ${ }^{2}$
}

Received: 21 July 2020 / Revised: 22 September 2020 / Accepted: 29 September 2020 / Published online: 7 October 2020

(c) Springer-Verlag GmbH Germany, part of Springer Nature 2020

\begin{abstract}
Objectives Evidences from either small series or spontaneous reporting are accumulating that SARS-CoV-2 involves the Nervous Systems. The aim of this study is to provide an extensive overview on the major neurological complications in a large cohort of COVID-19 patients.

Methods Retrospective, observational analysis on all COVID-19 patients admitted from February 23rd to April 30th, 2020 to ASST Papa Giovanni XXIII, Bergamo, Italy for whom a neurological consultation/neurophysiological assessment/neuroradiologic investigation was requested. Each identified neurologic complication was then classified into main neurologic categories.

Results Of 1760 COVID-19 patients, 137 presented neurologic manifestations that manifested after COVID-19 symptoms in 98 pts and was the presenting symptom in 39. Neurological manifestations were classified as: (a) cerebrovascular disease [53 pts (38.7\%)] including 37 ischemic and 11 haemorrhagic strokes, 4 transient ischemic attacks, 1 cerebral venous thrombosis; (b) peripheral nervous system diseases [31 (22.6\%)] including 17 Guillain-Barrè syndromes; (c) altered mental status [49 (35.8\%)] including one necrotizing encephalitis and 2 cases with RT-PCR detection of SARS-Cov-2 RNA in CSF; (d) miscellaneous disorders, among whom 2 patients with myelopathy associated with Ab anti-SARS-CoV-2 in CSF. Patients with peripheral nervous system involvement had more frequently severe ARDS compared to patients with cerebrovascular disease $\left(87.1 \%\right.$ vs $42 \%$; difference $=45.1 \% 95 \%$ CI $\left.42.0-48.2 ; \chi^{2}=14.306 ; p<0.0002\right)$ and with altered mental status $(87.1 \%$ vs $55.6 \%$; difference $=31.5 \% 95 \%$ CI $\left.27.5-37.5 \% ; \chi^{2}=7.055 ; p<0.01\right)$.

Conclusion This study confirms that involvement of nervous system is common in SARS-CoV-2 infection and offers clinicians useful information for prevention and prompt identification in order to set the adequate therapeutic strategies.
\end{abstract}

Keywords COVID-19 - Infectious diseases $\cdot$ Cerebrovascular diseases $\cdot$ Peripheral neuropathies $\cdot$ Altered mental status

Maria Sessa

msessa@asst-pg23.it

1 University of Milano-Bicocca, Milan, Italy

2 Department of Neurology, Papa Giovanni XXIII Hospital, Piazza OMS 1, 24127 Bergamo, Italy

3 Department of Neuroradiology, Papa Giovanni XXIII Hospital, Bergamo, Italy

4 Department of Neurology, Cremona Hospital, Cremona, Italy

5 Department of Laboratory Medicine, Papa Giovanni XXIII Hospital, Bergamo, Italy

\section{Introduction}

In December 2019, an outbreak caused by a novel coronavirus (2019-nCoV), now named severe acute respiratory syndrome coronavirus 2 (SARS-CoV-2), occurred in China and has rapidly spread all over the world causing a pandemic. The disease caused by SARS-CoV-2 was named COVID-19 [1]. In Europe, the first case was reported in the Lombardy region. Although soon after all Italian regions reported patients with COVID-19, the highest number of cases was in Eastern Lombardy [2], specifically in the Bergamo's 
province with 11,313 confirmed COVID-19 patients up to April 30th 2020 [3].

The typical spectrum of disease severity of SARS-CoV-2 infection is highly variable, ranging from asymptomatic carriers to severe acute respiratory distress syndrome (ARDS) leading to death. With the increasing number of confirmed cases and the accumulating clinical data, it is now well established that, in addition to the predominant respiratory symptoms, a significant proportion of patients has neurological manifestations [4-12]. Despite available studies have drawn attention to the neurological component of SARS-CoV-2 infection and provide detailed description of the neurological complications, they still have limitations. Data were collected either from limited or selected cohorts, and in the only nation-based study, case notification was on voluntary basis; diagnosis was mainly based on patient's description as cerebrospinal fluid analysis and instrumental examinations were avoided in order to reduce the risk of cross infection [11]. Moreover, scarce information regarding the impact of the neurologic involvement on clinical outcomes are available.

Here, we describe the neurologic manifestations observed in 137 patients among 1760 patients with laboratory or radiology-confirmed diagnosis of COVID-19, admitted to the ASST Papa Giovanni XXIII in Bergamo, in the epicentre of Italian pandemic, between February 23rd and April 30th, 2020. We provide an extensive overview on the different neurological complications involving both Central (CNS) and Peripheral (PNS) Nervous System, and on their impact on outcome.

\section{Methods}

\section{Study design, setting and patients selection}

The present study is a retrospective, observational analysis conducted at ASST Papa Giovanni XXIII on all adult patients of both sexes, with confirmed COVID-19 disease, admitted from February 23rd to April 30th, 2020. Our hospital was designated to treat patients with SARSCoV-2 infection. Within a few days from the beginning of the outbreak, $65 \%$ of beds were reconverted to COVID-19 patients and $70 \%$ staff doctors, regardless of their specialty, were redeployed to COVID-19 Units. We evaluated all neurological consultations/neurophysiological assessments/ neuroradiologic investigations requested for COVID-19 patients during hospitalization. We included in our analysis the following main diagnosis/syndromes: (a) cerebrovascular diseases (CVD) including ischemic and haemorrhagic stroke, transient ischemic attack, and cerebral venous thrombosis, (b) altered mental status including encephalitis/ meningitis/meningo-encephalitis, (c) peripheral nervous system disorders including Guillain-Barrè syndromes (GBS), Critical Illness Myopathy and Neuropathy (CRIMYNE), plexopathies and peripheral polyneuropathies. A few additional patients presenting symptoms/signs that did not fit these categories were classified as miscellaneous.

Wherever possible, informed consent was collected verbally. However, in most cases, due to the inability to provide informed consent by the patient or the inability to collect it in compliance with the contagion prevention measures, the principle of secondary use of data was used in accordance with art. 28, paragraph 2, letter b) of the November 20th, 2017 law, n. 167, included in the legislative decree 196/03 of art. 110-bis. The Institutional Review Board at ASST Papa Giovanni XXIII provided approval for the study $(257 / 2020,13 / 5 / 2020)$.

\section{Data collection}

Demographic (age, sex), comorbidities (hypertension, diabetes, dyslipidemia, atrial fibrillation, peripheral artery disease, cardiac disease or CVD, malignancy, dementia and lung disease), onset of neurological symptoms (before, concomitant, after COVID-19 onset), severity of ARDS according to the Horowitz index, clinical, laboratory and imaging data were extracted from medical records using a standardized anonymized data collection form by study physicians (NR, MS, SG).

\section{COVID-19 diagnosis}

COVID-19 diagnosis was confirmed: (1) by real-time reverse-transcriptase polymerase-chain-reaction (RT-PCR) on nasopharyngeal specimens [13]; or (2) by RT-PCR on bronchoalveolar lavage (BAL) obtained by bronchoscopy in case of high clinical suspicion of SARS-CoV-2 infection and negative test results on at least two nasopharyngeal swabs performed at least $24 \mathrm{~h}$ apart; or (3) in the presence of characteristic radiological interstitial pneumonia associated with typical symptoms (fever, dry cough, dyspnea), even with negative RT-PCR, with no other possible aetiologic explanation.

\section{Neurological evaluations and procedures}

The Neurology Department was reconverted to NeuroCOVID with 10 neurologists specifically dedicated and supported by a team of infectious disease specialists, pneumologists and intensivists. In addition, 5 other staff neurologists were dedicated to the Emergency Department and consultations in all the other COVID wards. Patients complaining of neurological symptoms were evaluated by means of standard neurological examinations, neurophysiological exams including electroencephalography 
(EEG), Evoked Potentials (EP), Electroneurographic and Electromyographic Recordings (ENG-EMG), and Brain Computerized Tomography (CT) and/or Magnetic Resonance (MR) according to standard care. Cerebrospinal Fluid (CSF) examination, when performed, included protein and glucose levels, cell count, cytological evaluation, research for bacteria and common neurotropic viral agents (Herpes Simplex 1-2, Varicella Zoster, Cytomegalovirus, Epstein Barr and Human Herpes Virus 6) and for SARSCoV-2 genome by Real-time PCR, Cobas ${ }^{\circledR}$ SARS-CoV-2 Test (Roche) and by GeneFinder COVID-19 (Elitech Group). $\mathrm{Ab}$ anti-SARS-CoV-2 on serum and CSF were tested by VivaDiagTM Covid-19 IgM/IgG immunochromatographic assay from VivaChekTM Biotech (China), performed according to manufacturer's instructions.

\section{Statistical analysis}

Frequencies were compared by means of the $\chi^{2}$ test with Yates' correction. Continuous variables were compared by means of the Wilcoxon rank sum test. Because of multiple comparisons, the significance level was set at 0.01 .

\section{Results}

From February 23rd to April 30th, a total of 1760 COVID19 patients were admitted to ASST Papa Giovanni XXIII and either discharged (1650) or still hospitalized (110). Among them, 137 (7.8\%) developed symptoms/signs of
CNS/PNS involvement. Table 1 shows the observed neurological complications. The more represented neurological manifestations fell into the following three categories: CVD (53 patients; 38.7\%), PNS diseases (31 patients; 22.6\%), altered mental status (49 patients; $35.8 \%$ ).

Their average age was $64.9 \pm 14.0$ (range $30-95$ years); $34 \%$ of the patients were female. The most frequent comorbidity was hypertension (in 83 pts; 60.6\%); diabetes was present in 24 pts (17.5\%), dyslipidemia in 31 (22.6\%), cardiovascular disease in 31 (22.6\%), malignancy in 13 (9.5\%), pulmonary disease in $21(15.3 \%)$, and previous neurological disease in 35 (25.5\%). The average number of comorbidities was 2.8 .

In 114 patients (83.2\%), diagnosis of SARS-CoV-2 infection was made by RT-PCR from pharyngeal swab; in the remaining $23(16.8 \%)$ patients with a negative swab, diagnosis was formulated in the presence of characteristic radiological interstitial pneumonia associated with typical symptoms, in some cases confirmed by positive BAL.

$\mathrm{PaO}_{2} / \mathrm{FiO}_{2}$ ratio was available at admission in $134 / 137$ pts. Sixty-nine patients $(51.5 \%)$ had a moderate or severe ARDS $\left(\mathrm{PaO}_{2} / \mathrm{FiO}_{2}\right.$ ratio lower than 200) according to the Horowitz index. Fifty-five patients $(40.1 \%)$ required admission to intensive care unit (ICU). ICU admission was significantly more frequent in patients with a moderate/ severe lung injury compared to those with a non-severe respiratory distress $(71.0 \%$ vs $7.7 \%$; diff. $63.3 \%$-95\% CI $\left.49.3-77.3 ; \chi^{2}=53.181 ; p<0.0001\right)$.

The neurological complication was the presenting symptom in 39 patients, 27 of whom never manifested fever and
Table 1 Observed neurological complications

\begin{tabular}{lllll}
\hline Neurological complications & $N(\%)$ & & \\
\cline { 2 - 5 } & All patients $(N=137)$ & $N$ of females $(\%)$ & Average age & $\begin{array}{l}\text { Average } n \text { of } \\
\text { comorbidities }\end{array}$ \\
\hline Cerebrovascular diseases & $53(38.7 \%)$ & $20(37.7 \%)$ & 68.6 & 3.1 \\
Ischemic stroke & $37(27.0 \%)$ & $15(40.5 \%)$ & 70.3 & 3 \\
Haemorrhagic stroke & $11(8 \%)$ & $4(36.3 \%)$ & 65.9 & 3.5 \\
Transient ischemic attacks & $4(2.9 \%)$ & $1(25 \%)$ & 63.5 & 3.4 \\
Cerebral venous thrombosis & $1(0.7 \%)$ & $0(0 \%)$ & 55 & 3 \\
Peripheral neuropathies & $31(22.6 \%)$ & $6(19.3 \%)$ & 56.3 & 2.1 \\
Guillain-Barrè syndrome & $17(12.4 \%)$ & $4(23.5 \%)$ & 55.6 & 2.2 \\
Critical illness polyneuropathy & $9(6.6 \%)$ & $1(1.1 \%)$ & 60.7 & 1.7 \\
Others & $5(3.6 \%)$ & $1(20.0 \%)$ & 54.2 & 3.6 \\
Altered mental status & $49(35.8 \%)$ & $17(34.7 \%)$ & 65.6 & 2.7 \\
Encephalitis & $5(3.6 \%)$ & $1(40 \%)$ & 66 & 2.4 \\
Myelitis & $2(1.4 \%)$ & $0(0 \%)$ & 64.5 & 3.5 \\
Headache & $3(2.2 \%)$ & $1(33.3 \%)$ & 61.5 & 0.7 \\
Seizures & $10(7.3 \%)$ & $3(30.0 \%)$ & 64.4 & 2.9 \\
Syncope & $3(2.2 \%)$ & $2(66.7 \%)$ & 72.6 & 3.7 \\
Movement disorders & $7(5 \%)$ & $3(42.8 \%)$ & 70.3 & 3.8 \\
Other & $5(3.6 \%)$ & $1(20.0 \%)$ & 61.2 & 4.6 \\
\hline
\end{tabular}


respiratory symptoms. In 98 pts, the neurologic complication manifested after a median of 16 days (range 1-64 days) from the onset of other clinical symptoms of COVID-19. Among these patients, 24 (24.5\%) developed the neurological complication within 7 days from the clinical manifestations of COVID-19; 21 (21.4\%) between 8 and 14 days, 15 (15.3\%) between 15 and 21 days, $8(8.2 \%)$ between 22 and 28 days and 30 (30.6\%) after 28 days. Twenty-one (70\%) of the patients with onset beyond 4 weeks were admitted to ICU and neurologic findings were recorded when sedation and neuromuscular blockade were withheld.

On April 30th 46/137 patients were still hospitalized. In the remaining 91 pts, mean $\pm \mathrm{SD}$ length of stay was $17.6 \pm 14.4$ days. Overall mortality rate during hospitalization was $29.7 \%$.

\section{Cerebrovascular disease}

Fifty-three patients (38.7\%) manifested CVD: 37 ischemic strokes $(27 \%), 4$ transient ischemic attacks $(2.9 \%), 11$ haemorrhagic complications ( $8 \%$ ), and 1 cerebral venous thrombosis $(0.7 \%)$ (Table 1$)$.

Their average age was $68.6 \pm 13.6$ (range 31-95 years), 20 patients $(37.7 \%)$ were female. Five patients had pulmonary thromboembolism. D-Dimer values were available for 33 CVD patients, and resulted increased in all cases [average value $8120 \mathrm{ng} / \mathrm{mL} \pm 11,056.8$ (range 539-35,000 ng/mL)].

There was a trend versus higher mortality in patients with CVD when compared to patients with PNS involvement (38.5\% vs $\left.11.8 \% ; \chi^{2}=4.511 ; p<0.05\right)$, but not when compared to patients with altered mental status (38.5\% vs $28.1 \% ; \chi^{2}=0.899$; NS). However, in the multivariable analysis that included age as a covariate, mortality was not significantly associated with any neurological group (data not shown).

\section{Clinical vignette}

A 66-year-old woman with a history of hypertension, ischemic cardiomyopathy, mechanical aortic valve replacement in anticoagulant therapy, and recent hospitalization for SARS-CoV-2 infection was readmitted to the Emergency Department (ED) for acute onset of left sensorimotor hemiparesis and dysarthria (NIHSS 6). Pharyngeal swab for SARS-CoV-2 was negative. Chest radiography showed evidence of previous bilateral interstitial pneumonia. Brain CT scan was normal; CT-angiography revealed a thrombus in her basilar apex. Intravenous alteplase was administered followed by mechanical thrombectomy with excellent angiographic (TICI 3) and clinical outcome (NIHSS 0). The following day, for the acute onset of left hemiplegia, hypoesthesia and dysarthria, a brain and angio-CT was repeated showing occlusion of the M1 segment of right MCA. Thrombectomy was successfully performed. An additional pharyngeal swab for SARS-CoV2 was positive. Cardiological investigations were unremarkable. The patient was discharged home at day 20 fully recovered.

\section{Altered mental status}

Forty-nine patients manifested disturbances of vigilance and/or consciousness. All performed either CT scan or brain MRI. Twenty-one pts underwent CSF analysis, in all of whom SARS-CoV-2 RT-PCR was performed. Based on clinical characteristics, CSF data and neuroimaging, we concluded with the diagnosis of encephalitis in 5 patients, among whom one HSV1-related, one necrotizing encephalitis, and two patients with detection of SARSCoV-2 by RT-PCR in CSF.

\section{Clinical vignette}

A otherwise healthy 60 -year-old man presented to ED because of headache and confusion. He left the hospital from the waiting room, and was later found wandering and confused. On readmission, he was afebrile and eupneic, with prominent agitation followed by a generalized tonic-clonic seizure. Chest radiography was normal. Blood tests revealed elevated levels of D-dimer, fibrinogen, LDH, IL-6, and C-reactive protein; blood cell counts were normal. Electroencephalogram and brain CT scan were unremarkable. CSF analysis showed normal protein level and cell count; culture was sterile; RT-PCR was negative for common neurotropic virus. RT-PCR for SARS-CoV-2 resulted positive both on pharyngeal swab and CSF. Hydroxychloroquine ( $200 \mathrm{mg}$ twice daily) was started together with aripiprazole at a daily dose of $15 \mathrm{mg}$. The patient was discharged home at day 6 fully recovered.

\section{Peripheral nervous system}

31 patients (22.6\%) manifested PNS involvement: 17 GBS, 9 Critical Illness Myopathy and Neuropathy (CRIMYNE), 2 brachial plexopathies, and 3 peripheral polyneuropathies (PNP) (Table 1). All patients performed EMG-ENG studies. For the diagnosis of GBS, Hadden criteria were applied [14]. CSF analysis was generally avoided as the majority of the patients were treated with Low Molecular Weight Heparin at high doses in primary prevention of SARS-CoV-2 induced thrombophilia. It was performed in 10 patients ( 7 with GBS, 2 with CRIMYNE, and 1 with PNP).

Moderate/severe ARDS was significantly more frequent in patients with PNS involvement compared to patients with CVD (87.1\% vs $42 \%$; diff. $45.1 \%$ - $95 \%$ CI $24.4-65.8$; $\left.\chi^{2}=14.306 ; p<0.0002\right)$ and to patients with altered mental 
status $(87.1 \%$ vs $55.6 \%$; diff. $31.5 \%-95 \%$ CI $6.8-56.2$; $\left.\chi^{2}=7.055 ; p<0.01\right)$. Twenty-eight were admitted to ICU.

The EMG-ENG study confirmed the diagnosis of CRIMYNE in nine and of GBS in sixteen patients. Albumino-cytological dissociation was present in 5/7 patients with GBS. CSF RT-PCR for SARS-CoV-2 was performed and negative in $8 / 10$. In one pt the diagnosis of GBS was formulated on clinical ground and CSF results.

Thirteen GBS patients underwent a blink reflex test, which showed a demyelinating pattern in either the facial and/or the trigeminal nerve in all cases, suggesting a frequent cranial nerve involvement.

Mean length of stay was significantly longer in pts with PNS involvement compared with patients with CVD $(Z=3.085 ; p<0.002)$.

\section{Miscellaneous}

In addition, we observed 2 patients (1.4\%) with myelitis, 3 patients (2.2\%) complaining of headache, 10 patients $(7.3 \%)$ with seizures, and 7 patients $(5.1 \%)$ with movement disorders.

\section{Clinical vignettes}

Patient 1: A otherwise healthy 62-year-old man presented to ED complaining of back-pain irradiating to lower extremities, sensory changes, weakness, and constipation. Neurological examination showed bilateral leg motor weakness (grade 4/5 MRC scale) and diminished sensation below the T11 sensory dermatome. The tendon reflexes were normal bilaterally, with normal cutaneous plantar reflex. SARS-CoV-2 nasopharyngeal swab and thoracic CT scan were negative. Serology testing revealed the presence of IgG antibodies to COVID-19 (148 AU/mL). Brain MRI was normal; spine MRI showed diffuse degenerative changes.

Patient 2: A 66-year-old man had been in his usual health state until 45 days before admission, when fever, anosmia, and ageusia developed and lasted for 10 days. No further investigations had been performed and no treatment started. Two weeks later, he complained of walking disturbances and numbness involving both lower extremities. On admission, he had bilateral leg weakness (grade 4/5 MRC scale), with reduced sensation to touch and acroparesthesia. Deep tendon reflexes were brisk with bilateral distal clonus and normal cutaneous plantar reflex. A spastic paraparetic gait was observed. The nasopharyngeal swab for SARS-CoV-2 and the chest radiograph were negative. However, serology testing revealed the presence of IgG antibodies to COVID19 (88.6 AU/mL) and chest CT scan demonstrated small ground-glass opacities, suggestive of previous pneumonia. Brain MRI was normal; spine MRI showed diffuse degenerative changes, with a conglutinated appearance of the roots of the cauda, which present a slight hyperintense signal in $\mathrm{T} 2$ sequences.

In both patients, CSF analysis revealed normal cell count and slightly increased protein level; RT-PCR for bacteria, common neurotropic virus, and SARS-CoV-2 were negative; IgG anti-SARS-CoV-2 were positive. In both, EMG-ENG showed a reduction of maximal voluntary activity; SEP and MEP recorded from lower limbs showed a bilateral medullar conduction block of the long motor and sensitive pathways.

Therapy and outcome: Pat.1-The patient received methylprednisolone $1 \mathrm{gr}$ intravenously for 5 days, followed by immunoglobulins $(0.4 \mathrm{~g} / \mathrm{kg}$ die $)$. Because of clinical worsening, immunoglobulins were stopped after 3 days and plasma exchange started. Oral steroid treatment was tapered gradually. Pat 2-The patient received 5 days of methylprednisolone $1 \mathrm{~g}$ intravenously, followed by 3 rounds of plasma exchange. Oral steroid treatment was tapered gradually. At discharge to Rehabilitation Unit, both patients were able to ambulate without support, although with a paretic gait, with residual mild sensory deficits.

\section{Discussion}

Although respiratory distress is the most distinctive clinical picture of SARS-CoV-2 infection, neurologic manifestations have been described. However, available evidence derives mainly from scanty series [4], selected populations [5], or single case descriptions [6-10]. A larger study, launched at the beginning of April, has been recently published [11], which reports 153 cases prospectively notified through online portals developed across the principal UK neuroscience bodies. The national and interdisciplinary structure of the study permitted to capture both neurological and psychiatric diagnoses nation-wide. However, although physicians were permitted to notify retrospectively recent cases, the majority of neurological complications that occurred in March are likely to be missed. In addition, because of the clinical demands of the pandemic, awareness of the study was likely to be scarce and voluntary-based case notification underreported. Clinical, laboratory, and radiological findings of 43 cases of COVID-19 neurological disease from Queen Square Hospital have been recently published [12]. The spectrum of neurological syndromes was similar to what encountered in our population, apart from inflammatory syndromes. Contrary to Paterson's cohort, we did not observe any case of acute disseminated encephalomyelitis, despite the majority of patients with altered mental status performed brain MR (data not shown). Paterson's cases were discussed in the context of multidisciplinary team meeting, thus representing a bias towards more complex and severe cases.

Here we report unselected neurologic manifestations on the largest COVID-19 population ever described 
within a single hospital. Among 1760 patients with laboratory or radiology-confirmed diagnosis of COVID19 disease, consecutively admitted to the ASST PG23 in Bergamo from February 23rd to April 30th 2020, 137 patients (7.8\%) developed a neurologic complication. The incidence of neurologic events observed in our population is much lower than the incidence reported by Mao et al. [4]. However, in Mao series, data regarding neurologic complications are based on subjective descriptions derived from medical records, and include aspecific symptoms such as dizziness or nerve pain. On the opposite, we have included only neurologic complications documented by neurologic consultancy. In addition, we have extensively evaluated patients with neurologic involvement by appropriate instrumental tests. On the contrary, in available literature, instrumental evaluations were not systematically performed either to reduce the risk of cross infection, as in the Chinese report, or likely due to the well-known difficulties in transferring patients admitted in ICU for instrumental tests, as in Helmes et al's report [5].

The mean age of our patients was 64.9 years, higher than in Wuhan series. The mean age observed in our series is in line with that reported from a large cohort of COVID19 patients admitted to ICUs in Lombardy [15], and likely reflects the higher median age of the Italian population. On the opposite, it is lower than the mean age of UK cases where, according to Authors, an older population could be overrepresented [11]. It is worth noting that more than $50 \%$ of our patients were below 65 years of age. This observation should alert physicians that neurologic involvement is not a exclusive prerogative of the older population.

In our cohort, neurologic event was the presenting manifestation in 39 pts, $70 \%$ of whom never developed other COVID-19 symptoms during hospitalization. This observation emphasizes that SARS-CoV-2 infection can manifest with predominant neurological symptoms and prompts the adoption of all specific measures to prevent contamination among patients and health professionals.

As in Varatharaj's study [11], the three principal groups of neurologic manifestations observed in our population were CVD, impaired consciousness and confusional states, and PNS involvement. Considering the entire COVID-19 population analysed (1760 pts in Bergamo cohort and 214 pts in Wuhan cohort), the prevalence of acute CVD was similar ( $3 \%$ vs $2.8 \%$ ). On the opposite, the prevalence of impaired consciousness and confusional states, as well as PNS involvement was inferior in our cohort when compared to that in Wuhan cohort ( $2.8 \%$ vs $7.5 \%$ and $1.8 \%$ vs $8.9 \%$ ). The observed differences are likely due to the fact that we considered only neurologic manifestations classified by means of appropriate laboratory and/or instrumental tests into specific nosographic categories.
It would be useful to compare the numbers of admissions for strokes, confusional states, and acute peripheral neuropathies in the same period of the previous year. Regarding stroke, the comparison is biased by the fact that, during pandemic, Lombardy region redesigned regional networks for acute conditions, centralizing stroke management in a few hubs. Diagnosis of confusional states and consciousness impairment are poorly traced in electronic records, making comparison impossible. On the contrary, it is worthwhile to note that we observed a dramatic increase in GBS diagnosis, when comparing with the equivalent period of the previous year (17 vs 3 ).

Mean lag time from COVID-19 symptom onset to neurologic manifestations in our population was 20.5 days, with a median of 16 days. This observation is consistent with previous studies describing neurologic events during infections by other Coronaviruses, such as SARS and MERS [16-18]. On the contrary, Mao et al. [4] report that most neurologic manifestations occurred early at a median time of 1-2 days, but exclude from the analysis CVD and impaired consciousness, which are the main complications observed in our and Varatharaj's [11] cohorts. It is of note that, even if almost half of the complications occurs within the first 2 weeks, still a relevant percentage can manifest late in the course of the disease, $23.5 \%$ between 2 and 4 weeks and $30.6 \%$ beyond 4 weeks from COVID-19 symptom onset. It has to be underlined that, among patients with detection of neurological signs beyond 4 weeks, 21 patients $(75 \%)$ were admitted to ICU, and neurologic findings were recorded when sedation and neuromuscular blockade were withheld; in eight of them a diagnosis of GBS was confirmed by EMGENG analysis. Considering that response to immunoglobulin treatment shortens recovery and improves disability score when administered within the early stage of the disease [19], our observation is particularly relevant as it points out the need to monitor ICU COVID-19 patients either by means of sedation windows or instrumentally by neurophysiologic tests for the appearance of PNS disorders.

In our cohort, we describe two patients with altered mental status and two cases of myelitis with detection in CSF of SARS-CoV-2 RNA and IgG anti-SARS-CoV-2, respectively. Direct demonstration of the SARS-Cov-2 genome has been reported only in two patients presenting with acute encephalopathy from Japan [9] and China [10] respectively. Only one case of acute necrotizing myelitis in a COVID-19 patient has been reported so far. However, SARS-CoV-2 PCR in CSF was negative and CSF antibodies to Covid-19 were not tested [20]. An additional case presenting with acute flaccid paralysis of bilateral lower limbs and sphincter incontinence has been described. However, no CSF analysis nor spinal MRI were performed, making diagnosis of myelitis presumptive [21]. Our additional cases further support the neuroinvasive 
potential of SARS-CoV-2 and strongly suggest the need to systematically perform CSF examination with PCR for SARS-CoV-2 genome and search for Ab anti-SARS-CoV-2 in patients with symptoms related to CNS involvement, even in the absence of respiratory syndrome at clinical and instrumental evaluations.

\section{Limitations}

To the best of our knowledge, this is the first study with the aim of intercepting all neurological complications of SARS-CoV-2 infections occurred in the biggest COVID19 population ever reported. Nevertheless, it has several limitations. First of all, it is a retrospective study. However, thanks to the reorganization of the hospital, the redeployment of the neurologists in staff to COVID units, and the awareness of healthcare personnel on the involvement of NS during SARS-CoV-2 infection, we are pretty confident that we could intercept the majority of relevant neurologic complications. Moreover, we immediately designed a database where collecting demographic, clinical, and instrumental data of patients within an observational study approved by our Institutional Review Board.

As we did not include patients discharged by the ED, we might have lost some patients with mild neurological symptoms. However, we can speculate that patients with evolving neurological problems would have been readmitted to the ED and therefore captured later. Conversely, we might have lost patients who died in the ED for severe ARDS and concomitant neurologic involvement. It has to be said that we evaluated all brain CT scan requested for COVID-19 patients and therefore we should not have missed evident acute brain lesions.

Because of the overwhelming access of severely ill COVID-19 patients, especially in the first weeks of the pandemic, we did not trace records of ageusia and anosmia at onset of COVID-19 symptoms. This is a relevant limit as these symptoms may testify the involvement of the olfactory bulbs, considered one of the possible access route of the virus to CNS. To circumvent this limit, we are planning to include this item in the medical history record to be administered to the patients during follow-up visits, fully aware that we will miss the data in deceased patients.

Even if we have data on short-term outcome, we are lacking data on the medium term. To accomplish this aim, we have planned a follow-up out-patient service for clinical and instrumental evaluations of all COVID-19 patients discharged from ASST PG23, which includes neurologic evaluation at 3 and 6 months, together with neuropsychologic, neurophysiologic and neuroradiologic exams, when appropriate.

\section{Conclusion}

This study confirms that involvement of NS is common in SARS-CoV-2 infection and offers clinicians useful information either for prevention or to early intercept neurologic manifestations and set the adequate therapeutic strategies. During the current pandemic caused by a novel virus, the importance of sharing worldwide data from large populations is crucial to prepare the national health systems facing all the potential complications and saving the highest number of lives. In addition, a better knowledge on the epidemiology and relevance of neurologic involvement may shed lights on the pathophysiology of the disease, help in understanding the impact on the severity of respiratory distress as well as on long-term outcome, and contribute to the development of preventive strategies against viral entry into the CNS.

Acknowledgements The authors wish to acknowledge Dr. M. Rizzi, Dr. A. Tebaldi, Dr. L. Soavi, Dr. F. Zoboli, Dr. M. Cerrone, and all infectious disease specialists; Dr. M. Molteni and Dr. R. Del Fabbro; Dr. L.F. Lorini, Dr. P. Gritti, Dr. F. Fabretti, Dr. L. Grazioli, Dr. F. Ferri and all intensivists; prof. F. Di Marco and all pneumologists; the nursing staff of all the COVID-Units, for their unremitting and professional care of patients. This work is dedicated to all the patients who lost their life because of COVID-19 pandemic and to their families.

Author contributions MS had full access to all of the data in the study and takes responsibility for the integrity of the data and the accuracy of the data analysis. Concept and design: NR, MS. Acquisition of the data: EA, DA, VB, GC, BC, MZC, CF, BF, SG, MG, SLG, TP, SQ, RR, MCS, MS, MV, EV. Analysis and interpretation of data: NR, MS. Laboratory analyses: AC, MA. Drafting of the manuscript: NR, MS. Critical revision of the manuscript for important intellectual content: NR, MS, BC. Statistical analysis: BC.

\section{Compliance with ethical standards}

Conflicts of interest The authors declare that they have no competing interest.

Ethical standard statement Wherever possible, informed consent was collected verbally. However, in most cases, due to the inability to provide informed consent by the patient or the inability to collect it in compliance with the contagion prevention measures, the principle of secondary use of data was used in accordance with art. 28, paragraph 2, letter b) of the November 20th, 2017 law, n. 167, included in the legislative decree 196/03 of art. 110-bis. The Institutional Review Board at ASST Papa Giovanni XXIII provided approval for the study (257/2020, $13 / 5 / 2020$ ). All procedures followed were in accordance with the ethical standards of the responsible committee on human experimentation (institutional and national) and with the Helsinki Declaration of 1975, as revised in 2000 .

\section{References}

1. Liu YC, Kuo RL, Shin SR (2020) COVID-19: the first documented coronavirus pandemic in history. Biomed J. https://doi. org/10.1016/j.bj.2020.04.007 (Available online 5 May 2020) 
2. Livingston E, Bucher K (2020) Coronavirus disease 2019 (COVID-19) in Italy. JAMA 323(14):1335. https://doi. org/10.1001/jama.2020.4344

3. http://www.salute.gov.it/portale/news/p3_2_1_1_1.jsp?lingu $\mathrm{a}=\mathrm{italiano} \& \mathrm{menu}=$ notizie $\& \mathrm{p}=$ dalministero\&id $=4648$

4. Mao L, Jin H, Wang M et al (2020) Neurologic manifestations of hospitalized patients with coronavirus disease 2019 in Wuhan, China. JAMA Neurol. https://doi.org/10.1001/jamaneurol .2020 .1127 (published online ahead of print, 2020 Apr 10)

5. Helms J, Kremer S, Merdji H et al (2020) Neurologic features in severe SARS-CoV-2 infection. N Engl J Med 382(23):2268-2270. https://doi.org/10.1056/NEJMc2008597

6. Toscano G, Palmerini F, Ravaglia S et al (2020) Guillain-Barré syndrome associated with SARS-CoV-2. N Engl J Med. https ://doi.org/10.1056/nejmc2009191 (published online ahead of print, 2020 Apr 17)

7. Zhao H, Shen D, Zhou H, Liu J, Chen S (2020) Guillain-Barré syndrome associated with SARS-CoV-2 infection: causality or coincidence? Lancet Neurol 19(5):383-384. https://doi. org/10.1016/S1474-4422(20)30109-

8. Alberti P, Beretta S, Santoro PM et al (2020) Guillain Barré syndrome associated with SARS-CoV-2. Neurol Neuroimmunol Neuroinflamm 7(4):e741. https://doi.org/10.1212/NXI.0000000000 000741

9. Moriguchi T, Harii N, Goto J et al (2020) A first case of meningitis/encephalitis associated with SARS-Coronavirus-2. Int J Infect Dis 94:55-58. https://doi.org/10.1016/j.ijid.2020.03.062

10. Xiang P, Xu XM, Gao LL et al (2020) First case of 2019 novel coronavirus disease with encephalitis. ChinaXiv:T202003.00015

11. Varatharaj A, Thomas N, Ellul MA et al (2020) Neurological and neuropsychiatric complications of COVID-19 in 153 patients: a UK-wide surveillance study. Lancet Psychiatry. https://doi. org/10.1016/S2215-0366(20)30287-X

12. Paterson RW, Brown RL, Benjamin L et al (2020) The emerging spectrum of COVID-19 neurology: clinical, radiological and laboratory findings. Brain. https://doi.org/10.1093/brain/awaa240

13. Cheng MP, Papenburg J, Desjardins M et al (2020) Diagnostic testing for severe acute respiratory syndrome-related coronavirus 2. Ann Intern Med 172(11):726-734. https://doi.org/10.7326/ M20-1301
14. Hadden RD, Cornblath, Hughes RA et al (1998) Electrophysiological classification of Guillain-Barré syndrome: clinical associations and outcome. Plasma Exchange/Sandoglobulin GuillainBarré Syndrome Trial Group. Ann Neurol 44(5):780-788. https:// doi.org/10.1002/ana.410440512

15. Grasselli G, Zangrillo A, Zanella A et al (2020) Baseline characteristics and outcomes of 1591 patients infected with SARSCoV-2 admitted to ICUs of the Lombardy Region, Italy. JAMA 323(16):1574-1581. https://doi.org/10.1001/jama.2020.5394 (published online ahead of print, $2020 \mathrm{Apr} 6$ )

16. Umapathi T, Kor AC, Venketasubramanian N et al (2004) Large artery ischaemic stroke in severe acute respiratory syndrome (SARS). J Neurol 251(10):1227-1231. https://doi.org/10.1007/ s00415-004-0519-8

17. Arabi YM, Harthi A, Hussein J et al (2015) Severe neurologic syndrome associated with Middle East respiratory syndrome corona virus (MERS-CoV). Infection 43(4):495-501. https://doi. org/10.1007/s15010-015-0720-y

18. Algahtani H, Subahi A, Shirah B (2016) Neurological complications of middle east respiratory syndrome coronavirus: a report of two cases and review of the literature. Case Rep Neurol Med 2016:3502683. https://doi.org/10.1155/2016/3502683

19. Hughes RA, Raphaël JC, Swan AV, van Doorn PA (2006) Intravenous immunoglobulin for Guillain-Barré syndrome. Cochrane Database Syst Rev (1):CD002063. https://doi.org/10.1002/14651 858.cd002063.pub3

20. Sotoca Javier, Rodríguez-Álvarez Yensa (2020) COVID-19-associated acute necrotizing myelitis. Neurol Neuroimmunol Neuroinflamm 7(5):e803. https://doi.org/10.1212/NXI.000000000000080 3

21. Zhao K, Huang J, Dai D, Feng Y, Liu L, Nie S (2020) Acute myelitis after SARS-Cov-2 infection: a case report. Medrxiv. https ://doi.org/10.1101/2020.03.16.20035105 